\title{
Low-temperature control of nanoscale molecular dynamics
}

\author{
Per Hyldgaard \\ Department of Applied Physics, Chalmers University of Technology and Göteborg University \\ S-41296 Göteborg, Sweden \\ E-mail: hyldgaar@fy.chalmers.se \\ Received February 23, 2001
}

\begin{abstract}
A novel in situ probe of the nanoscale molecular dynamics of organic-molecule and fullerene-tube nanostructures is proposed. General and consistent results for the nonlinear-current coupling to the nanostructure excitations are presented to document a frequency-selective electrostatic control of this current stimulation and optimal operation as a local source of current-induced molecular excitations $\Omega_{i}$. The control is possible for temperatures $T \ll \Omega_{i}$. Finally, it is explained in detail how Raman measurements of this molecular dynamics would probe the nanoscale excitations within organic and fullerene nanostructures under non-linear transport conditions.
\end{abstract}

PACS: 85.65.+h, 85.30.Mn, 73.40.Gk

A successful future molecular-electronics technology requires an understanding of the fascinating nanoscale molecular devices themselves, of their nonlinear and interacting transport properties, and of the current-induced molecular dynamics. Fullerene tubes (nanotubes) [1] and organic molecules [2], which assemble between metal contacts to form organic nanostructures, offer interesting candidates for such a molecular-transport program. These organic nanostructures and the fullerene tubes implement, for example, the molecule resonant-tunneling diode [3], the single-Bucky-ball $\left(\mathrm{C}_{60}\right)$ transistor [4], and the nanotube field-effect transistor [5], which achieves room-temperature operation and a nanoscale-feature size in all but the transport direction. Fullerene-tube heterostructures can be identified by experimentally observed kinks [6] and permit additional nanoscale molecular-electronic devices by combining sections of different local chirality [7] and thus a different nature of electron conduction [8]. The experimental selection of single-kink heterojunctions produce the nanotube equivalent of current-rectifying diodes [6]. A corresponding selection [6], and/or proposed engineering [9], of double-kink nanotube samples produces either the nanotube quantum dot [10] or the robust, i.e., temperature- and scattering-insensitive, nanotube resonant-tunneling transistor [11], which achieves a nanoscale-feature size in all dimensions.
Nanostructure device robustness is of central importance as, e.g., experiments on single-Bucky-ball transistors [4] and on current-induced atom/molecule manipulation [12], document significant molecular excitation induced by the nonlinear transport [13]. The coupling to this molecular excitation can even provide novel transport mechanisms as in the electron shuttle [14]. A molecular-electronics program must characterize devices both in terms of the nonlinear molecular current and in terms of the nonlinear-transport coupling to the molecule-structural dynamics - the molecular excitation.

Here I document frequency-selective electrostatic control of the current-induced molecular excitations and propose a probe of this local nanostructure dynamics. The control and suggested molecular probe work for characteristic excitation frequencies $\Omega_{i}=\Omega_{0,1 \ldots}$ that are larger than the temperatures $T$ and would operate at the relevant nonequilibrium device condition, i.e., with the nonlinear transistor current enabled. For use in the molecular-dynamics probe, furthermore detail optimal operation as a strong frequency-selective source of current-induced molecular excitations at the large frequencies $\Omega_{i} \gg T_{\text {room }}$ that characterize the local fullerenetube nanostructure dynamics [15]. The suggested nanostructure probe extends an earlier proposal by Narayanamurti [16] that used a burst of incoherent (acoustic) phonons to identify and map defects deep 
inside semiconductor heterostructures. Here, instead, I propose (1) to exploit the nonlinear nanostructure transport conditions for a direct in situ and controlled excitation of the relevant high-energy vibrations $\Omega_{i}-\Omega_{0,1, \ldots}$ and (2) to use surface-enhanced Raman spectroscopy $[15,17]$ to measure the resulting molecular excitation $\delta N_{\mathrm{vib}}\left(\Omega_{i}\right)$, establish the associated decay, $1 / \tau_{i}$, and thus probe the density of material defects [16], the mutual coupling between such vibrations, and the intrinsic nature of the excitation (phonon) propagation [18,19].

Figure 1 illustrates a pair of resonant-tunneling systems that could produce a strong source of molecular excitations and hence the proposed nanostructure-dynamics probe. The upper scheme involves an organic nanostructure [3] (ORTN) - an organic molecule assembled between and connected both mechanically and electrically to the source and drain metal contact through the synthesisized inclusion of sulphur atoms $\mathrm{S}$. The lower scheme involves a double-kink nanotube resonant-tunneling heterostructure (NRTH) [11], in which metallic nanotube leads (grey tubes) connect to the metal contacts (wedges), e.g., scanning-tunneling microscope tips. In both schemes the central barrier region traps a single resonant level of energy $E_{\text {orb }}$ and connected by tunneling rates $\Gamma_{L / R}$ to the surrounding metal leads or contacts. A close metal gate, e.g., another metallic nanotube (grey ring at potential $\Phi_{\text {gate }}$ ) adjusts $E_{\text {orb }}\left(\Phi_{\text {gate }}\right)$ and enables a gate-controlled resonant-tunneling transport [4,11, 20,21].

The proposed nanostructure-dynamics probe exploits this gate-control of the nanostructure tunnel-

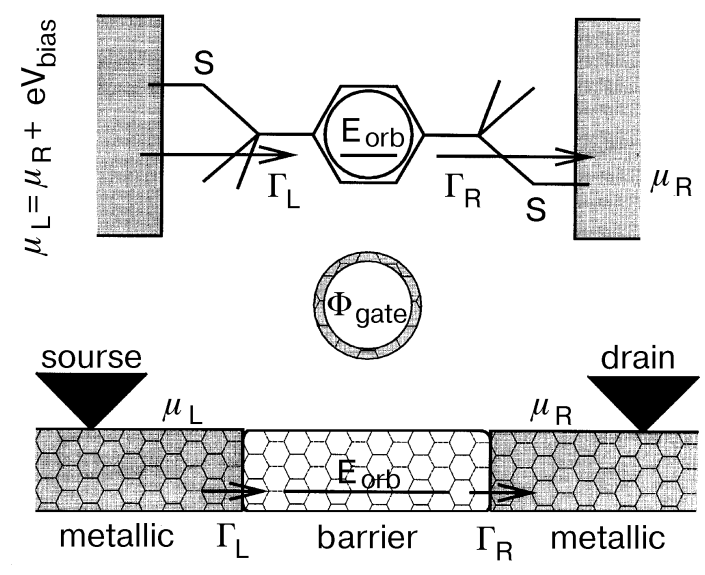

Fig. 1. Schematics of transport and local vibration-source realizations as organic resonant-tunneling nanoparticle (top panel) or as resonant-tunneling nanotube heterostructure (bottom panel). A metal gate (grey ring) at voltage $\Phi_{\text {gate }}$ controls the current and molecular excitation in either nanostructures by adjusting the resonant-level energy position $E_{\text {orb }}\left(\Phi_{\text {gate }}\right)$. ing transport by a selective optimization of the current-induced molecule stimulation. To document the suggested operation, this paper (i) provides a conserving nonequilibrium Green function calculation $[13,22]$ of the current-induced spontaneous emission (net absorption) rates $R_{\mathrm{sp}}\left(E_{\text {orb }} ; \Omega_{i}\right)$ $\left[R_{\mathrm{ab}}\left(E_{\text {orb }} ; \Omega_{i}\right)\right]$ as a function of $E_{\text {orb }}\left(\Phi_{\text {gate }}\right)$ and at a set of characteristic excitation frequencies; (ii) details how phase-space restrictions that rest on the Pauli exclusion principle permit a frequency-selective gate control of $R_{\mathrm{sp}, \mathrm{ab}}\left(E_{\mathrm{orb}} ; \Omega_{i}\right)$; and (iii) identifies nonequilibrium tunneling conditions that can maximize and/or inhibit the resulting effective current excitation $\delta N_{\mathrm{vib}}\left(\Omega_{i}\right)$. At a given gate voltage and thus resonant- energy position $E_{\text {orb }}\left(\Phi_{\text {gate }}\right)$, determine an excess nanostructure vibrational population [23]:

$$
\begin{aligned}
& \frac{1}{\tau_{i}} \delta N_{\mathrm{vib}}\left(E_{\mathrm{orb}}\left(\Phi_{\text {gate }}\right) ; \Omega_{i}\right)=\left[R_{\mathrm{sp}}\left(E_{\text {orb }} ; \Omega_{i}\right)-\right. \\
& \left.-R_{\mathrm{ab}}\left(E_{\text {orb }} ; \Omega_{i}\right) \delta N_{\mathrm{vib}}\left(E_{\text {orb }}\left(\Phi_{\text {gate }}\right) ; \Omega_{i}\right)\right] .
\end{aligned}
$$

The local electrostatic-field control $E_{\text {orb }}\left(\Phi_{\text {gate }}\right)$ $[4,11,20]$ can, for example, produce a strong frequency-selective) burst of nanostructure excitations $\delta N_{\text {vib }}\left(\Omega_{0}\right)$. Simultaneous Raman measurements [15] of the strength of the anti-Stokes Raman signal at nanostructure-vibration frequency $\Omega_{0}$ can thus determine the decay $1 / \tau_{0}$ of this excess population [17] $\delta N_{\mathrm{vib}}\left(\Omega_{0}\right)$ and probe intrinsic mechanisms $[16,18,19]$ affecting the nanoscale molecular dynamics.

\section{Electrostatic control of resonant-tunneling transport}

A theory description of the electrostatic gate control $E_{\text {orb }}\left(\Phi_{\text {gate }}\right)$ exists for both the organic nanostructure [20] and the fullerene heterostructure [11] scheme (Fig. 1, upper and lower schemes). In both transport schemes this local electrostatic control permits current-switch and transistor effects $[4,11,20]$ in which transport is focused onto a single molecular level. Such nanostructure transistors improve the semiconductor resonant-tunneling transistor design [21] through a dramatic miniaturization to nanoscale dimensions. For a calculation of the noninteracting resonant- tunneling transport in an organic-nanostructure transistor, refer to the analysis in Ref. 20. For a nonequilibrium Green function $[13,22]$ calculation of the interacting transport in the nanotube heterostructure transistor (Fig. 1, lower scheme) refer to Ref. 11. Before reporting calculations of gate control in the current-induced 


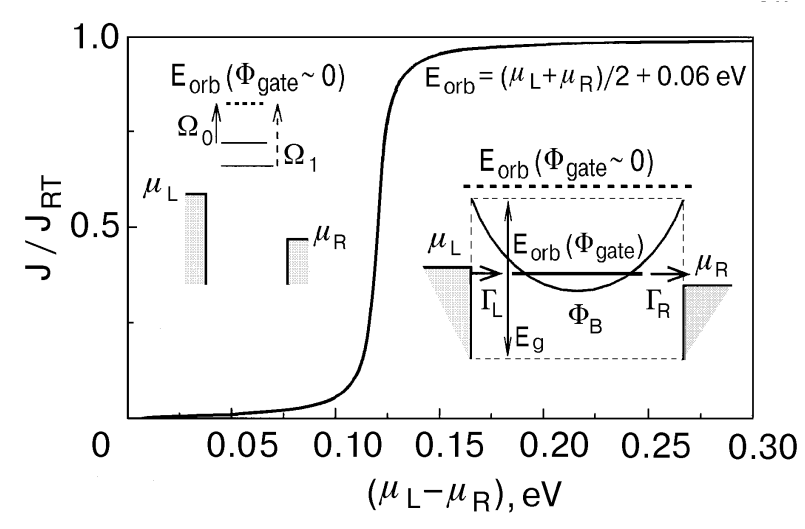

Fig. 2. Gate control of nonequilibrium resonant-tunneling current $J<J_{R T}$. The insert panels contrast transport conditions at (i) $\Phi_{\text {gate }} \sim 0$, when the molecular gab $E_{g}$ forces a resonancelevel energy position $E_{\text {orb }}\left(\Phi_{\text {gate }} \sim 0\right)$ (dashed line in right insert) and vibration satellites $E_{\text {orb }}\left(\Phi_{\text {gate }} \sim 0\right)-\Omega_{0,1}$ (left insert) far above the lead chemical potentials $\mu_{L / R} ;$ And at (ii) $\Phi_{\text {gate }} \sim 2 \mathrm{~V}$, when the adjusted electron potential $\Phi_{B}$ (solid curve in right insert) positions $E_{\text {orb }}\left(\Phi_{\text {gate }}\right) \approx\left(\mu_{L}+\mu_{R}\right) / 2$ (solid line). The main panel assumes such a fixed $E_{\text {orb }}\left(\Phi_{\text {gate }}\right)$ : a moderate bias $\mu_{L}-\mu_{R} \approx 0.3 \mathrm{eV}$ then saturates the current $J \approx J_{R T}$.

molecular excitation, however, I summarize the description of the important tunneling-transport mechanisms $[11,20]$.

Figure 2 illustrates the electrostatic-gate effects on the nonlinear resonant-tunneling current. A finite applied bias $e V_{\text {bias }}$ maintains the left and the right metallic-nanotube leads at the different chemical potentials $\mu_{L}$ and $\mu_{R}=\mu_{L}-e V_{\text {bias }}$, respectively. The main panel in Fig. 2 assumes that a finite gate voltage $\Phi_{\text {gate }}$ maintains a fixed resonantlevel position $E_{\text {orb }}\left(\Phi_{\text {gate }}\right)=\left(\mu_{L}+\mu_{R}\right) / 2+0.06 \mathrm{eV}$ and it documents how the application of a moderate bias can then saturate a significant resonant-tunneling current

$$
e J<e J_{R T}=e\left(\frac{4 \Gamma_{L} \Gamma_{R}}{\Gamma_{L}+\Gamma_{R}}\right) \sim\left\{\begin{array}{l}
1 \mathrm{nA} \text { for ORTN }, \\
5 \mu \mathrm{A} \text { for NRTH } .
\end{array}\right.
$$

The current is characterized by the resonance width $\Gamma=\Gamma_{L}+\Gamma_{R}$. At low temperatures this tunneling current $[13,22,24]$

$$
J\left[E_{\text {orb }}\left(\Phi_{\text {gate }}\right) ; \mu_{L / R}\right]-J_{R T}\left[P_{\text {occ }}^{\mu_{L}}\left(E_{\text {orb }}\right)-P_{\text {occ }}^{\mu_{R}}\left(E_{\text {orb }}\right)\right]
$$

results as a difference between contributions

$$
P_{\text {occ }}^{\mu}\left(E_{\text {orb }}\right)=\left[\frac{1}{2}+\frac{1}{\pi} \arctan \left(\frac{\mu-E_{\text {orb }}}{\Gamma}\right)\right]
$$

evaluated at $\mu=\mu_{L}$ and $\mu_{R}$, respectively. I focus on the NRTH transport realizations where it is possible to achieve $\Gamma \sim 10 \mathrm{meV}$ [11].

The pair of inset diagrams illustrate the gate operation and contrast the transport conditions in the absence and presence of a finite voltage $\Phi_{\text {gate }}$ applying to the metal gate. At $\Phi_{\text {gate }} \sim 0$ (left inset) the molecular gap $E_{g}(\gtrsim 1 \mathrm{eV}$ for the previously investigated NRTH transistor [11]) forces a resonance-level energy position $E_{\text {orb }}\left(\Phi_{\text {gate }} \sim 0\right) \approx$ $\approx \mu_{L}+E_{g} / 2$ (dashed line in right inset). No current results, since both $E_{\text {orb }}$ and the vibration satellites [13] $E_{\text {orb }}\left(\Phi_{\text {gate }} \sim 0\right)-\Omega_{i=0,1}$ remain far above the chemical potentials of the leads. However, a voltage $\Phi_{\text {gate }} \sim 2 \mathrm{~V}$ suppresses the electron potential $\Phi_{B}$ (solid curve, right inset), within the fullerene barrier and adjusts the resonance-level position $E_{\text {orb }}\left(\Phi_{\text {gate }}\right) \approx\left(\mu_{L}+\mu_{R}\right) / 2$ (solid line) to enable the tunneling processes (arrows). Below I assume a fixed bias $e V_{\text {bias }}=300 \mathrm{meV}$ and use the resonant-level gate control $E_{\text {orb }}\left(\Phi_{\text {gate }}\right)$ also to optimize the current stimulation of molecular excitations.

\section{The molecular excitations}

For the NRTH it is relevant to consider currentinduced excitation at energies $\Omega_{i}=100-200 \mathrm{meV}$ (800-1600 $\left.\mathrm{cm}^{-1}[15]\right)$. I concentrate on a pair of high-energy modes, at assumed frequencies $\Omega_{1(0)}=$ $=200 \mathrm{meV}(120 \mathrm{meV})>>\Gamma$ and describe the current stimulation at zero temperature to illustrate my results and predictions.

The top and middle panels in Fig. 3 contrast the independent gate-voltage control of the electron-vibration interaction effects in $R_{\mathrm{sp}}$ and $R_{\mathrm{ab}}$ for a pair of vibration energies $\Omega_{0}$ (black curve) and $\Omega_{1}$ (dashed curve). The gate control is implicit as the gate voltage $\Phi_{\text {gate }}$ adjusts the resonant level energy position $E_{\text {orb }}\left(\Phi_{\text {gate }}\right)-\mu_{L}$. The fixed applied bias satisfies $2 \Omega_{1}>e V_{\text {bias }}>2 \Omega_{0}$ and $e V_{\text {bias }}>\Omega_{1}$. The documented current-excitation gate control arises within the region $\mu_{L}>E_{\text {orb }}>\mu_{R}$ (identifed by vertical lines), where the electrostatic gate enables a strong resonant-tunneling current, Eq. (3). The excitation transition rates are illustrated for equal electron tunneling rates $\Gamma_{L}=\Gamma_{R}$.

I determine the magnitude and gate dependence of the current-induced molecular-excitattion transition rates $R_{\mathrm{sp}}$ and $R_{\mathrm{ab}}$ through a separate nonequilibrium Green function calculation similar to that for the nonequilibrium defluctuations (shot noise) [25]. The calculation involves a determination of the nonequilibrium density-correlation components: 


$$
\begin{aligned}
& \Pi_{r}^{0}(\omega)=-i \int_{0}^{\infty} d t^{\prime} \exp \left(i \omega t^{\prime}\right)\left\langle\left[n\left(t+t^{\prime}\right) n(t)\right]\right\rangle, \\
& \Pi_{<}^{0}(\omega)=-\int_{-\infty}^{\infty} d t^{\prime} \exp \left(i \omega t^{\prime}\right)\left\langle n\left(t+t^{\prime}\right) n(t)\right\rangle,
\end{aligned}
$$

where $n$ denotes the electron density at the resonance and where the notations and conventions introduced in Ref. 13 are followed. The retarded correlation component includes a commutator [.,.] and defines the frequency shift [13] and vibration decay [26] due to the electron-vibration (phonon) interaction. Here I evaluate both components (5), (6) out of equilibrium to establish the current-induced excitation level, Eq. (1).

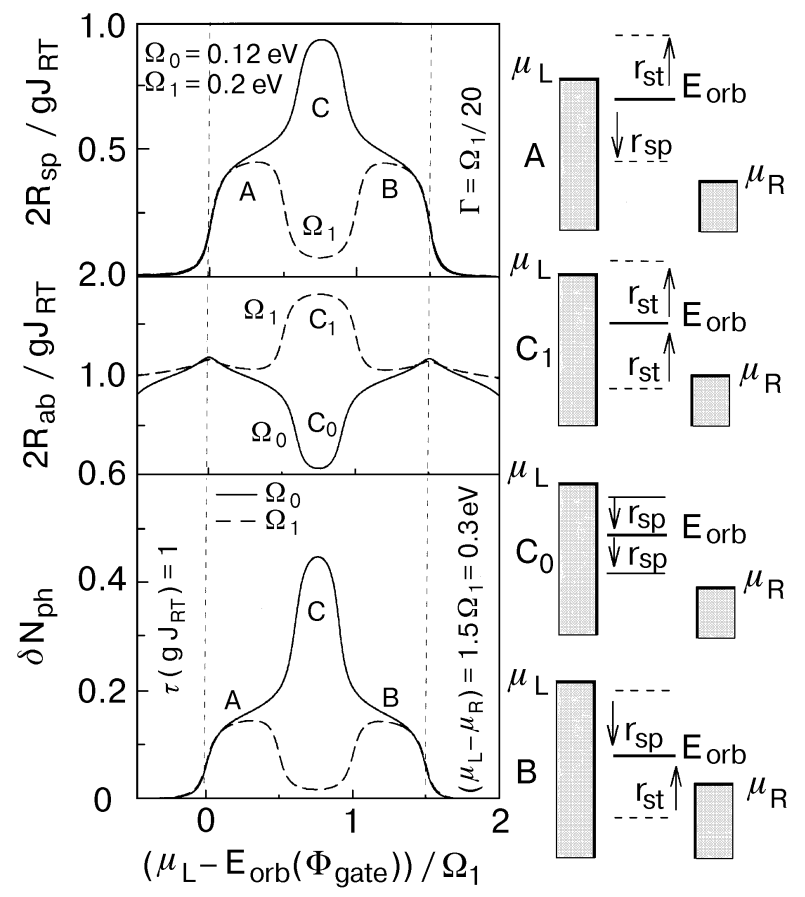

Fig. 3. Frequency selective current-stimulation of molecular vibrations. The left-most top and middle panels contrast the gate variation of the spontaneous phonon emission rate $R_{\mathrm{sp}}$ and net absorption rate $R_{\mathrm{ab}}$ at two frequencies $\Omega_{0,1}$. Both rates are proportional to $J_{R T}$ and the electron-vibration coupling constant $g$. The gate variation is implicit and defined through $E_{\text {orb }}\left(\Phi_{\text {gate }}\right)$. The set of four right-most schematics illustrates the Pauli exclusion mechanism responsible for the frequency-selective control. The set of downward (upward) arrows labeled by $r_{\mathrm{sp}}\left(r_{\mathrm{ab}}\right)$ identify inelastic tunneling events that contribute to the spontaneous emission (to the net stimulated absorption). Finally, the left-most bottom panel contrasts the current-induced increase in excitation level $\delta N_{\mathrm{vib}}\left(\Omega_{0}\right)$ (solid curve) and $\delta N_{\mathrm{vib}}\left(\Omega_{0}\right)$ (dashed curve) for a given intrinsic vibration decay time $\tau$.
The spontaneous vibration emission rate $R_{\mathrm{sp}}$ is to lowest order in the dimensionless electron-vibration coupling strength $g$ [13], given by the < correlation component, Eq. (6). The rate is proportional to $J_{R T}$ but limited to the availability of phase space [27]:

$$
\begin{gathered}
\frac{R_{\mathrm{sp}}\left[E_{\mathrm{orb}}\left(\Phi_{\text {gate }}\right) ; \Omega_{i}\right]}{g J_{R T}}=\frac{\left[-\Omega^{2} \Pi_{<}^{0}\left(\Omega_{i}\right) / 2\right]}{J_{R T}}= \\
=\frac{\Theta\left(\mu_{L}-\mu_{R}-\Omega_{i}\right) \Omega_{i}^{2}}{4\left(\Omega_{i}^{2}+4 \Gamma^{2}\right)} \times \\
\times\left[\Delta P_{\text {occ }}\left(E_{\text {orb }} ; \Omega_{i}\right)+\Delta P_{\log }\left(E_{\text {orb }} ; \Omega_{i}\right)\right] .
\end{gathered}
$$

A significant variation and structure arises in the vibration emission (7) when $\Omega_{i}>>\Gamma$, condition relevant for the local molecular modes of the NRTH. The spontaneous vibration emission is then dominated by the simple phase-space measure

$$
\begin{gathered}
\Delta P_{\text {occ }}\left(E_{\text {orb }} ; \Omega_{i}\right)=\left[P_{\text {occ }}^{\mu_{L}}\left(E_{\text {orb }}\right)-P_{\text {occ }}^{\mu_{R}}\left(E_{\text {orb }}-\Omega_{i}\right)\right]+ \\
+\left[P_{\text {occ }}^{\mu_{L}}\left(E_{\text {orb }}+\Omega_{i}\right)-P_{\text {occ }}^{\mu_{R}}\left(E_{\text {orb }}\right)\right]
\end{gathered}
$$

while the logarithmic correction term $\Delta P_{\log }$ can be ignjred [28]. The phase-space measure (8) is specified by the nonequilibrium contributions (4) at the resonance level and at the vibration satellites $E_{\text {orb }} \pm \Omega$.

The net vibration absorption at $\Omega_{i} \gg \Gamma$ is given by [29]

$$
\begin{gathered}
\quad \frac{R_{\mathrm{ab}}\left[E_{\mathrm{orb}}\left(\Phi_{\mathrm{gate}}\right) ; \Omega_{i}\right]}{g J_{R T}}=\frac{\Omega^{2}\left[-\operatorname{Im} \Pi_{r}^{0}\left(\Omega_{i}\right)\right]}{J_{R T}} \approx \\
\approx \frac{\Gamma \Omega_{i}^{2}}{4\left(\Omega_{i}^{2}+4 \Gamma^{2}\right)}\left(\Gamma _ { R } ^ { - 1 } \left[P_{\mathrm{occ}}^{\mu_{L}}\left(E_{\text {orb }}-\Omega_{i}\right)-P_{\text {occ }}^{\mu_{L}}\left(E_{\text {orb }}+\Omega_{i}\right]+\right.\right. \\
\left.+\Gamma_{L}^{-1}\left[P_{\text {occ }}^{\mu_{R}}\left(E_{\text {orb }}-\Omega_{i}\right)-P_{\text {occ }}^{\mu_{R}}\left(E_{\text {orb }}+\Omega_{i}\right)\right]\right)
\end{gathered}
$$

This rate is defined by a phase-space measure which, in contrast to Eq. (8), involves differences of contributions, Eq. (4), evaluated at the same chemical potential (at $\mu_{L}$ or $\mu_{R}$ ). Thus it is possible to achieve an important independent control of $R_{\text {sp }}\left[E_{\text {orb }}\left(\Phi_{\text {gate }}\right) ; \Omega_{i}\right]$ and $R_{\text {ab }}\left[E_{\text {orb }}\left(\Phi_{\text {gate }}\right) ; \Omega_{i}\right]$.

The Pauli-exclusion principle explains the phasespace limitations on the current-induced spontaneous and net absorption, Eqs. (8) and (9). The four right-most panels, $A, C_{0}, C_{1}$, and $B$, in Fig. 3 illustrate the set of different transport conditions 
(all with $J \sim J_{R T}$ ) which characterize the gate-control regimes identified in the left set of panels.

The presence of downward arrows $r_{\mathrm{sp}}$ identify conditions when the current flow can stimulate a spontaneous vibration emission, as specified by the phase-space measure, Eq. (8). In region $A(B)$ this emission arises when an electron tunnels into $E_{\text {orb }}$ (into $E_{\text {orb }}+\Omega_{i=0,1}$ ) but leaves at energy $E_{\text {orb }}-\Omega_{i=0,1}$ (at $E_{\text {orb }}$ ). For an applied bias which satisfies $2 \Omega_{1}>e V_{\text {bias }}>\Omega_{1}$ (panel $C_{1}$ ) neither type- $A$ nor type- $B$ vibration-emission processes are possible for local mode $\Omega_{1}$. However, both types of spontaneous emission processes remain possible for a vibrational mode at $\Omega_{0}<e V_{\text {bias }} / 2\left(\right.$ panel $\left.C_{0}\right)$.

The presence of upward arrows $r_{\mathrm{ab}}$ instead identifies conditions for a net current-induced absorption $R_{\mathrm{ab}} \neq 0$. In section $A(B)$ a net absorption arises, when the electron enters at $E_{\text {orb }}$ (at $E_{\text {orb }}$ $-\Omega_{i=0,1}$ ) but leaves at $E_{\text {orb }}+\Omega_{0,1}$ (at $E_{\text {orb }}$ ). Tuning $E_{\text {orb }}$ to the central region $C$ causes an enhanced absorption for mode $\Omega_{1}$, as both type- $A$ and type- $B$ absorption processes become possible (panel $C_{1}$ ). However, for the lower mode at $\Omega_{0}<e V_{\text {bias }} / 2$ I find an effective cancellation (panel $C_{0}$ ) as the energies $E_{\text {orb }}$ and $E_{\text {orb }} \pm \Omega_{0}$ all carry a partial electron occupation and thus produces a vanishing net absorption rate, $R_{\mathrm{ab}} \rightarrow 0$.

\section{Frequency-selective molecular-vibration stimulation}

The lower-left panel in Fig. 3 documents how an optimization of current-induced molecular excitation is possible. The panel contrasts the calculated gate-variation of the increase in the molecular excitation level, Eq. (1), $\delta N_{\mathrm{vib}}\left(\Omega_{0}\right)$ and $\delta N_{\mathrm{vib}}\left(\Omega_{1}\right)$, and details methods to enhance the current stimulation of mode $\Omega_{0}$ at the expense of mode $\Omega_{1}>\Omega_{0}$. Such selective excitation is possible even when $e V_{\text {bias }}>\Omega_{1}>>\Gamma$, and arises when $2 \Omega_{1}>e V_{\text {bias }}>$ $>2 \Omega_{0}$ and $E_{\text {orb }}$ is tuned to region $C\left(E_{\text {orb }} \approx\left(\mu_{L}+\right.\right.$ $\left.+\mu_{R}\right) / 2$ ). These nonequilibrium transport conditions simultaneously minimize $R_{\mathrm{st}}\left(\Omega_{1}\right)$ towards zero and maximize the ratio $R_{\mathrm{st}}\left(\Omega_{0}\right) / R_{\mathrm{ab}}\left(\Omega_{0}\right)$ to extinguish $\delta N_{\mathrm{vib}}\left(\Omega_{1}\right)$ and dramatically enhance the lower-frequency current stimulation $\delta N_{\mathrm{vib}}\left(\Omega_{0}\right)$.

\section{The molecular-dynamics source and probe.}

The lower-left panel in Fig. 3 also details the suggested operation as a molecular-excitation source. The panel documents a crisp electro- static gate control for the current-stimulation $\delta N_{\mathrm{vib}}\left(\Omega_{1}\right)$ which arises through an adjustment of the resonantlevel energy position $E_{\text {orb }}\left(\Phi_{\text {gate }}\right)$ [11]. This implicit gate control permits a switch between enabling and disabling the current-stimulation (1). Such operation can produce a frequency-selective molecular-vibration source and even a strong nonequilibrium burst $\delta N_{\text {vib }}\left(\Omega_{0}\right)>>0$ of high-energy nanostructure vibrations.

Nanoscale molecular-dynamics probing is then possible with simultaneous Raman measurements of the anti-Stokes signal at $\Omega_{0}$, because the anti-Stokes strength is directly sensitive [17] to the excess molecular-excitation burst $\delta N_{\text {vib }}\left(\Omega_{0}\right)>>0$. Such Raman measurement can through Eq. (1) determine the decay $1 / \tau_{i}$ that characterize these nanostructure molecular excitations and thus probe mechanisms $[16,18,19]$ which help determine the intrinsic nanoscale molecular dynamics. The suggested in situ molecular-dynamics probe could realize an important strong testing of our theoretical descriptions for both the nanostructure atomic configurations $[10,11,30]$ and for the current-induced structural dynamics [4,12-14].

In summary, I have suggested a novel in situ probe of the nanoscale molecular dynamics of organic-molecule and fullerene-tube heterostructures. General nonequilibrium Green function results for the current coupling to local nanostructure excitations were presented to document a frequency-selective electrostatic control and optimal operation as a necessary current-excitation source. Raman measurements of the anti-Stokes signal can then permit an in-situ probe of the local nanostructure molecular dynamics at nonequilibrium conditions.

I thank R. Shekhter, B. I. Lundqvist, M. Persson, and M. Jonson for valuable discussions. Financial support from the Swedish Foundation for Strategic Research (SSF), through the Materials Consortium no. 9, is gratefully acknowledged.

1. S. Iijima, P. M. Ajayan, and T. Ichihashi, Phys. Rev. Lett. 69, 3100 (1992); C. Dekker, Phys. Today 52 (5), 22 (1999).

2. See, e.g., C. Zhou, M. R. Deshpande, M. A. Reed, L. Jones II, and J. M. Tour, Appl. Phys. Lett. 71, 611 (1997); L. Jones II, D. L. Pearson, J. S. Schumm, and J. M. Tour, Pure Appl. Chem. 68, 145 (1996); J. M. Tour, Chem. Rev. 96, 537 (1996).

3. J. Chen, M. A. Reed, A. M. Rawlett, and J. M. Tour, Science 286, 1550 (1999).

4. H. Park, J. Park, A. K. L. Lim, E. H. Anderson, A. P. Alivisatos, and P. L. McEuen, Nature 407, 57 (2000).

5. S. J. Tans, A. R. M. Verschueren, and C. Dekker, Nature 393, 49 (1998).

6. Z. Yao, H. W. C. Postma, L. Balents, and C. Dekker, Nature 402, 273 (1999); Article supplement in Nature's archive documents observation and wiring/measurement of also double-kink nanotube heterostructures.

7. R. Saito, M. Fujita, G. Dresselhaus, and M. S. Dresselhaus, Phys. Rev. B46, 1804 (1992)

8. C. T. White and J. W. Mintmire, Nature 394, 30 (1998). 
9. D. Orlikowski, M. B. Nardelli, J. Bernholc, and C. Roland, Phys. Rev. Lett. 83, 4132 (1999).

10. L. Chico, M. P. L. Sancho, and M. C. Munoz, Phys. Rev. Lett. 81, 1278 (1998)

11. P. Hyldgaard and B. I. Lundqvist, Solid State Commun. 116, 569 (2000).

12. B. C. Stipe, M. A. Rezaei, W. Ho, S. Gao, M. Persson, and B. I. Lundqvist, Phys. Rev. Lett. 78, 4410 (1997).

13. P. Hyldgaard, S. Hershfield, J. H. Davies, and J. W. Wilkins, Annals of Physics 236, 1 (1994); J. H. Davies, S. Hershfield, P. Hyldgaard, and J. W. Wilkins, Phys. Rev. B47, 4603 (1993).

14. L. Y. Gorelik, A. Isacsson, M. V. Voinova, B. Kasemo, R. I. Shekhter, and M. Jonson, Phys. Rev. Lett. 80, 4526 (1998).

15. K. Kneipp, H. Kneipp, P. Corio, S. D. M. Brown, K. Shafer, J. Motz, L. T. Perelman, E. B. Hanlon, A. Marucci, G. Dresselhaus, and M. S. Dresselhaus, Phys. Rev. Lett. 84, 3470 (2000); K. Kneipp, Y. Wang, H. Kneipp, L. T. Perelman, I. Itzkan, R. R. Dasari, and M. S. Feld, Phys. Rev. Lett. 78, 1667 (1997)

16. V. Narayanamurti, Science 213, 717 (1981).

17. A. Yariv, Quantum Electronics, John Wiley \& Sons, New York (1989), pp. 453-475; K. Kneipp, Y. Wang, H. Kneipp, I. Itzkan, R. R. Dasari, and M. S. Feld, Phys. Rev. Lett. 76, 2444 (11996).

18. S. G. Walkauskas, D. A. Broido, K. Kempa, and T. L. Reinecke, J. Appl. Phys. 85, 2579 (1999); P. Hyldgaard and G. D. Mahan, Thermal Conductivity 23, Technomic Publishing Company, Inc., Lancaster, Pennsylvania (1996), p. 172.

19. P. Hyldgaard and G. D. Mahan, Phys. Rev. B56, 10754 (1997).

20. M. Di Ventra, S. T. Pantelides, and N. D. Lang, Appl. Phys. Lett. 76, 3448 (2000).

21. M. A. Reed, W. R. Frensley, R. J. Matyi, J. N. Randall, and A. C. Seabaugh, Appl. Phys. Lett. 54, 1034 (1989).

22. S. Hershfield, J. H. Davies, and J. W. Wilkins, Phys. Rev. lett. 67, 3720 (1991); D. C. Langreth, 1975 NATO Advanced Study Institute on Linear and Nonlinear Electron
Transport in Solids, Antwerpen, 1975, B17, Plenum, New York (1976), p. 3.

23. Our results apply to linear order in the current and describe the stimulation of local high-energy nanostructure vibrations. Cases where the tunneling rate coincides with standing-wave resonances requires a treatment of also nonlinear excitation effects; See, e.g., Ref. 12.

24. R. Tsu and L. Esaki, Appl. Phys. Lett. 22, 562 (1973); M. Büttiker, Phys. Rev. Lett. 57, 1761 (1986)

25. J. W. Wilkins, S. Hershfield, J. H. Davies, P. Hyldgaard, and C. J. Stanton, Physica Scripta T42, 115 (1992); S. Hershfield, Phys. Rev. B46, 7061 (1992); P. Hyldgaard and S. Hershfield, unpublished.

26. B. Hellsing and M. Persson, Physica Scripta 29, 360 (1984).

27. The rate $R_{\mathrm{sp}}$ is just the excitation increase, $\delta N_{\mathrm{ph}}=\delta\left\langle b^{+} b\right\rangle$, as one enables the current-vibration coupling, $g>0$. I introduce $A_{0}(\omega) \equiv\left(2 \tau_{i}\right) /\left[1+\tau^{2}\left(\omega-\Omega_{i}\right)^{2}\right]$ as the unperturbed vibration-spectral function and find, to lowest order in $g$, the current stimulation $2 \delta N_{\mathrm{ph}} \approx g \Omega_{i}^{2} \int \Pi_{<}^{0}(\omega) A_{0}(\omega) d \omega$. The result Eq. (7) applies for $\tau_{i}^{-1}<<\Gamma$.

28. The logarithmic correction to the phase-space measure is given by $\Delta P_{\log }\left(E_{\text {orb }} ; \Omega_{i}\right)=\left(\Gamma / \pi \Omega_{i}\right) \sum_{\alpha=1,2} \ln \left(\Delta_{\alpha}^{2}+\Gamma^{2}\right)-\left(\Gamma / \pi \Omega_{i}\right) \times$ $\times \sum_{x=L, R} \ln \left[\left(\mu_{X}-E_{\text {orb }}\right)^{2}+\Gamma^{2}\right]$, where $\Delta_{1(2)}=\left(\mu_{L(R)}-E_{\text {orb }}-(+) \Omega_{i}\right) ;$ The prefactor $\left(\Gamma / \pi \Omega_{i}\right)$ ensures a smooth behavior at general $\Gamma / \Omega_{i}$ and a vanishing one, $\Delta P_{\log } \rightarrow 0$ at $\Gamma \ll \Omega_{i}$.

29. A small logarithmic correction to $R_{\mathrm{ab}}$ is given through the full nonequilibrium determination of $\Pi_{r}^{0}(\omega)$ [13]. I stress that $-\operatorname{Im} \Pi_{r}^{0} \geq 0$ and that single-resonance tunneling never produces coherent emission.

30. See, e.g., J. M. Seminario, A. G. Zacarias, and J. M. Tour, J. Am. Chem. Soc. 120, 3970 (1998); A. Rubio, D. SanchezPortal, E. Artacho, P. Ordejon, and J. M. Soler, Phys. Rev. Lett. 82, 3520 (1999). 